\title{
The application of a piezo-resistive cardiorespiratory sensor system in an automobile safety belt
}

\section{Syed Talha Ali Hadmani, Anura Fernando*, Parasd Potluri}

Textiles Research Group, School of Materials, The University of Manchester, Manchester, M13 9PL, UK; E-Mails: talha.hamdani@postgrad.manchester.ac.uk; anura.fernando-2@manchester.ac.uk; prasad.potluri@manchester.ac.uk

* Author to whom correspondence should be addressed; E-Mail: anura.fernando-2@manchester.ac.uk Tel.: +44-161-306-3753; Fax: +44-161-306-8804.

\begin{abstract}
Respiratory and heart failure are conditions that can occur with little warning and may also be difficult to predict. Therefore continuous monitoring of these bio-signals is advantageous for ensuring human health. The car safety belt is mainly designed to secure the occupants of the vehicle in the event of an accident. In the current research a prototype of safety belt is developed, which is used to acquire respiratory and heart signals, under laboratory conditions. The current safety belt is constructed using a copper ink based peizo-resistive material, which works on the piezo-resistive effect due to the pressure exerted on the sensor as a result of expansion of the thorax/abdomen area of the body for respiration and due to the principle of ballistocardiography in heart signal sensing. The research explains the science behind the construction of the safety belt and its integration into the safety belt.
\end{abstract}

Keywords: Woven; safety belt; cardiorespiratory signals; wearable sensor; piezo material

\section{Introduction}

The increased interest of researchers and industrialists in the field of wearable sensors has caused the work done in this area to gain a lot of importance in the last few decades [1]. Specifically, the capability to use these wearable sensors to monitor ubiquitously the health of patients over a long period of time has gained them much respect in the medical field. In the medical field these wearable sensors are used to provide real time patient medical feedback of possible forthcoming health threats to patient carers. Respiratory and heart failure are conditions that can occur with little warning and may also be difficult to predict. Therefore continuous monitoring of these bio-signals is advantageous for 
ensuring human health [2]. The diseases like sleep apnea syndrome and sudden infant death syndrome (sudden unexplained death before the age of one year) are also directly related to respiration and cardiac abnormalities respectively, requiring continuous and long term health monitoring $[3,4]$. When there is an abnormality in the function of the human body, the nervous system may cause an increase in the heart rate or respiration cycles. The blood pressure may increase as well, along with many other abnormal indications. If such abnormalities occur repeatedly in the body, it can affect the performance of the cardiac system, which can eventually present itself as a heart attack [5]. The respiration and heart rate also reflect the emotions and mental stresses a person experiences. One of the proactive approaches available to avoid such disease and to look after health in daily life is the ubiquitous monitoring of the human physiology.

The ubiquitous monitoring can be achieved by integrating a bio sensor into a portable device. Due to the importance of auto vehicles in everyday life, the integration of biosensors into the accessories/ upholstery of a car, namely the car seat belt or car seat cover can help ubiquitous monitoring. According to World Bank statistics [6], more than 50\% of the population in United Kingdom uses motor vehicles. Through surveys conducted in New South Wales, Australia it was found that $69 \%$ of the general population generally drive to work [7] indicating the capacity to which a bio-signal monitoring system in motor vehicles can influence the general health of the population.

Various studies have been conducted to capture the cardio respiratory signals using a wider range of wearable technologies. The very first study in the field of wearable technology for capturing the cardio respiratory signals was conducted by Gopalsamy, C., et al. in 1999 [8]. The main purpose of this study was the transmission of heart signals from a wearable shirt integrated with solid state sensors. The other technologies involve the use of LED and photodiodes [9], microwave Doppler techniques [10], ultrasonic sensors [11] optical fibers [12], piezoelectric [13] and piezo-resistive [14] response of a material etc. Out of these, piezo-resistive nonwoven fabric have been used as active electrodes for the measurement of bio-potential and respiratory signals [15]. In contrast, in the current research, a nonwoven fabric impregnated with a piezo-resistive ink has been used as the sensor material integrated into an automobile safety belt.

\section{Experimental Section}

The construction of an automobile safety belt integrated with sensors mainly consist of three manufacturing stages; safety belt weaving process, preparation of sensor and integration of the sensors into the safety belt. The safety belt was woven using a high performance narrow fabric weaving machine where the piezo-resistive sensors are integrated in to the safety belt as a post construction process. The conductive paths, made of electro-conductive yarn too were also created on either side of the sensor during the weaving process to acquire the electrical signals from them. The sensors in the current case were constructed out of nonwoven piezo-resistive material while electrodes made with silver knitted fabric, which were used to acquire the electrical signal resulting from the mechanical compression the sensor experiences. The knitted structure used in the knitted silver electrodes allow eleastic bidirectional movement in both warp and weft directions of the fabric making the structure 
suitable for applications experiencing either uni-directional or bidirectional deformation. The thickness of the silver knitted electrodes was found to be an important factor during the experiments since increased thickness in the sensor material showed that they tend to absorb the weak mechanical compression signals given out by BCG signals from the heart. Numbers of these sensors were integrated near the thorax and abdomen position of the prototype belt in order to ensure the capture of the best breathing and heart rate signals. The general positions of the sensors with respect the subject wearing this smart safety belt is shown in Figure 1.

Figure 1. Subject wearing the prototype safety belt

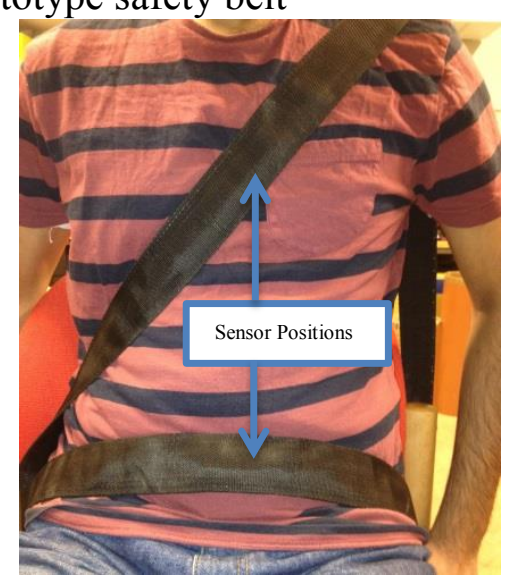

The characterization of sensors under mechanical load testing was carried out by the experimental setup shown in Figure 2. Whereas the cardio respiratory signals were acquired using an NI-9219 data acquisition module.

Figure 2. Experimental setup

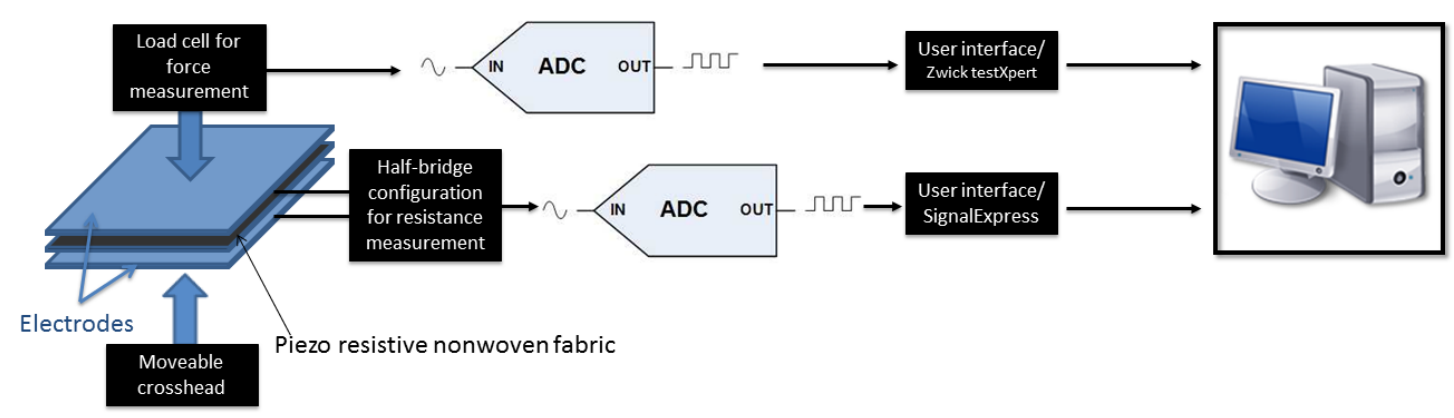

\section{Results and Discussion}

It can be seen from Figure 3 that the resistance of piezo-resistive sensors decreases sharply as the applied pressure is increased up to $20 \mathrm{~g} / \mathrm{cm}^{2}$ and after that the increase in pressure has a marginal effect on the change in resistance. In this case, the pressure of $20 \mathrm{~g} / \mathrm{cm}^{2}$ can be attributed as a critical pressure for this particular piezo-resistive non-woven sensor, where an appreciable change of resistance can be seen. 
Figure 3. Sensors characteristic curve

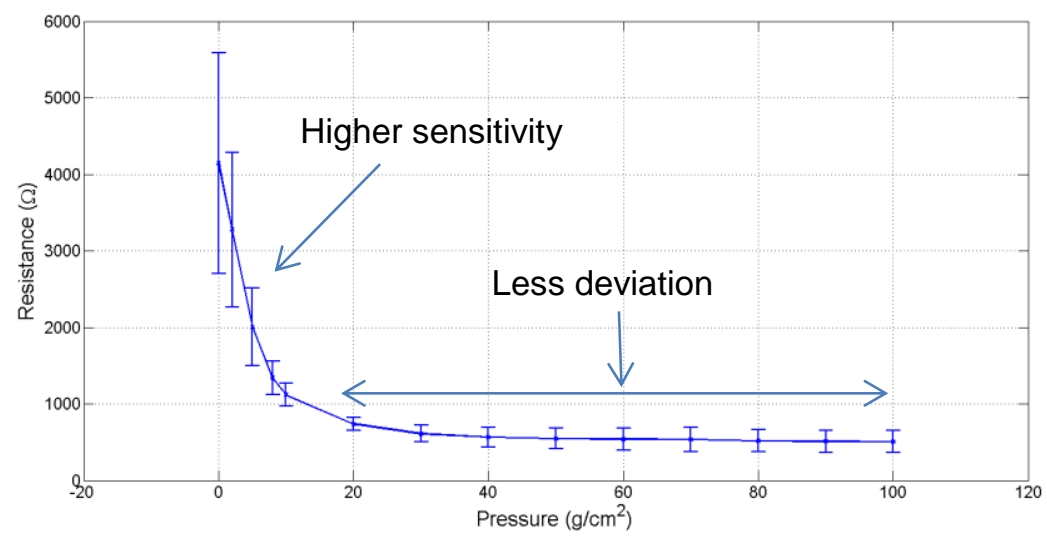

It was also observed that this sensor is responsive at very low pressure and capable of reacting to a weak signal due to a change of pressure by registering the peaks of it. However due to the nature of the piezo-resistive fabric material, the registered peaks do not give an absolute value of cardiorespiratory signals. Additionally, the results are dependent on the posture of the subject, proper placement of sensor and motion artefacts. It is also to be noted that, for efficient mechanical compressive signal acquisition, the belt needs to be wrapped on a curved area rather than a flat surface, in order for the belt stress in the longitudinal direction to create a stress in the transvers direction that is incident on the sensor. It was found that the sensor inside the belt experiences the pressure in the range of $20-30 \mathrm{~g} / \mathrm{cm}^{2}$ which was confirmed by using a Kikuhime pressure monitoring device.

The cardio respiratory signals achieved from the current prototype safety belt are shown in Figure 4 . It was observed that the sensor was capable of registering the respiration signals as shown in figure $4 \mathrm{a}$ whereas the capturing of heart signal using this piezo-resistive sensor was quite challenging. Even after applying a band pass filter, it was hard to differentiate between the noise and heart signals due to the similarity in the characteristics of both BCG signals and noise signals. In order to make sure the capturing of heart signal (rather than noise signals) using the piezo-resistive sensor, the heart signals from piezo-resistive sensor were acquired in synchronization with the BCG signals obtained from a standard BCG measuring device (biosignals plux). It was observed that the respiration signals obtained from the thorax and abdomen were quite smooth and the strength of the signal obtained from the sensor at abdomen was higher as compared to that of the sensor at thorax. This was mainly because of the larger volume of expansion of abdomen as compared to the volume of expansion of thorax. In the case of capturing the heartbeat, the results achieved with piezo-resistive sensors were similar to those presented by Paalasmaa, J. and M. Ranta [16]; the ideal heart beat would not have the same amplitude at every single heartbeat, rather it should be enveloped in a bell-like form. The heartbeat was also observed to contain some additive noise. It was observed that the heartbeat signals from piezo-resistive sensors can be acquired near the thorax position by holding the breath. However, as the sensibility of heartbeat is almost minimized near the abdomen it was near impossible to observe the heartbeat by the sensors placed at abdomen. 
Figure 4. Results from prototype of safety belt

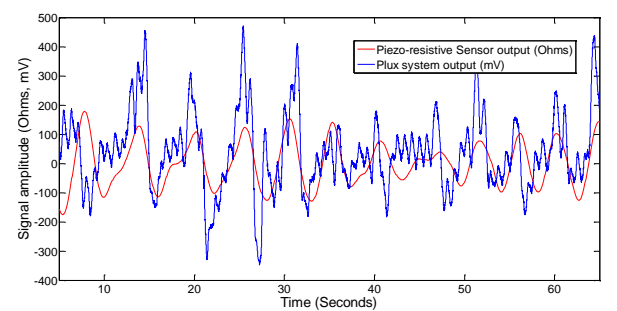

Breathing signals on thorax

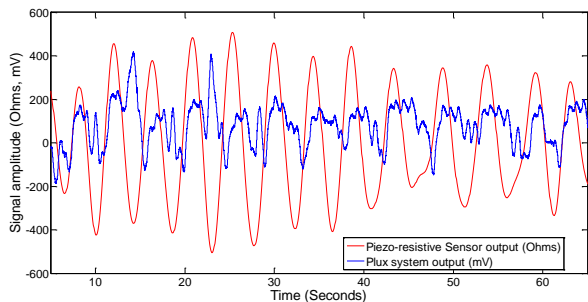

Breathing signals on abdomen

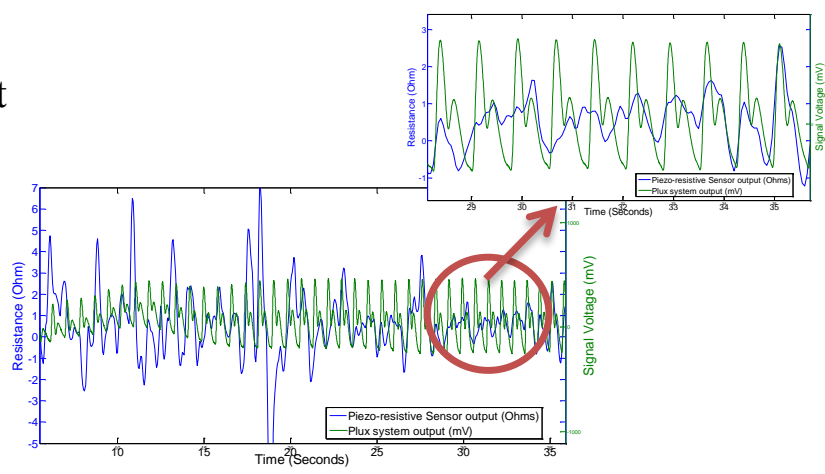

Heart rate signals on thorax

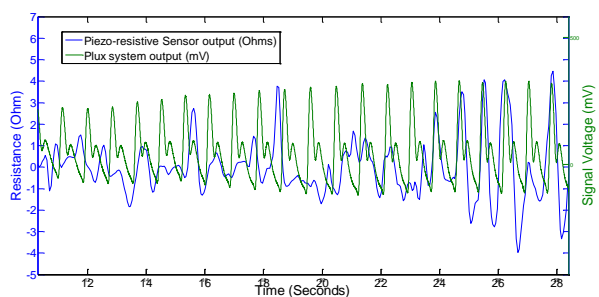

Heart rate signals on abdomen

The summary of results obtained from the prototype of the safety belt is tabulated in Table 1 .

Table 1. Strength of cardio respiration signals

\begin{tabular}{ccc}
\hline Position of sensor & Breathing signals $(\boldsymbol{\Omega})$ & Heart signals $(\boldsymbol{\Omega})$ \\
\hline Thorax & $200-400$ & $2-6$ \\
Abdomen & $700-900$ & n/a \\
\hline
\end{tabular}

\section{Conclusion}

In the current research work, the piezo-resistive sensor showed quite promising results for capturing the respiration signals. It was observed that the stable respiration signals were achieved from the abdomen of test subjects due to larger curved abdomen part as compared to the generally flat thorax. Capturing the heart signal with the current configuration of the sensor is challenging. However the heart beat can be detected near the thorax position with small signals amplitude. Further research is being carried out to improve the sensitivity of the sensor and high level filtering is carried out to complement the sensitivity of it in order to extract the heart rate.

\section{Acknowledgments}

The research was supported by the funding from the European Union Seventh Framework Programme (FP7/2007-2013) under grant agreement $n^{\circ}: 286265$

\section{Conflicts of Interest}

The authors declare no conflict of interest. 


\section{References and Notes}

1. Bonato, P., Wearable sensors/systems and their impact on biomedical engineering. Engineering in Medicine and Biology Magazine, IEEE 2003, 22, 18-20.

2. Witt, J.; Narbonneau, F.; Schukar, M.; Krebber, K.; De Jonckheere, J.; Jeanne, M.; Kinet, D.; Paquet, B.; Depre, A.; D"Angelo, L.T., et al., Medical textiles with embedded fiber optic sensors for monitoring of respiratory movement. Sensors Journal, IEEE 2012, 12, 246-254.

3. Niizeki, K.; Nishidate, I.; Uchida, K.; Kuwahara, M., Unconstrained cardiorespiratory and body movement monitoring system for home care. Med. Biol. Eng. Comput. 2005, 43, 716-724.

4. Moon, R.Y.; Fu, L.Y., Sudden infant death syndrome. Pediatrics in Review 2007, 28, 209-214.

5. Choi, S.; Jiang, Z., A novel wearable sensor device with conductive fabric and pvdf film for monitoring cardiorespiratory signals. Sensors and Actuators A: Physical 2006, 128, 317-326.

6. http://data.worldbank.org/, Motor vehicles (per 1,000 people). In 2009-2013, The World Bank Group.

7. Rajala, S.; Lekkala, J., Film-type sensor materials pvdf and emfi in measurement of cardiorespiratory signals - a review. Sensors Journal, IEEE 2012, 12, 439-446.

8. Gopalsamy, C.; Park, S.; Rajamanickam, R.; Jayaraman, S., The wearable motherboard ${ }^{\mathrm{TM}}$ : The first generation of adaptive and responsive textile structures (arts) for medical applications. Virtual Reality 1999, 4, 152-168.

9. Lei, W.; Lo, B.P.L.; Guang-Zhong, Y., Multichannel reflective ppg earpiece sensor with passive motion cancellation. Biomedical Circuits and Systems, IEEE Transactions on 2007, 1, 235-241.

10. Fletcher, R.R.; Kulkarni, S. In Clip-on wireless wearable microwave sensor for ambulatory cardiac monitoring, Engineering in Medicine and Biology Society (EMBC), 2010 Annual International Conference of the IEEE, Aug. 31 2010-Sept. 4 2010, 2010; pp 365-369.

11. Lanata, A.; Scilingo, E.P.; De-Rossi, D., A multimodal transducer for cardiopulmonary activity monitoring in emergency. Information Technology in Biomedicine, IEEE Transactions on 2010, 14, 817-825.

12. Nishyama, M.; Miyamoto, M.; Watanabe, K., Respiration and body movement analysis during sleep in bed using hetero-core fiber optic pressure sensors without constraint to human activity. Journal of biomedical optics 2011, 16, 017002-017002-017007.

13. Kärki, S.; Lekkala, J., A new method to measure heart rate with emfi and pvdf materials. Journal of Medical Engineering \& Technology 2009, 33, 551-558.

14. Jeong, J.W.; Jang, Y.W.; Lee, I.; Shin, S.; Kim, S., Wearable respiratory rate monitoring using piezo-resistive fabric sensor. In World congress on medical physics and biomedical engineering, september 7 - 12, 2009, munich, germany, Dössel, O.; Schlegel, W., Eds. Springer Berlin Heidelberg: 2009; Vol. 25/5, pp 282-284.

15. Kang, T.-H., Textile-embedded sensors for wearable physiological monitoring systems. NCSU Libraries: 2007; pp 11-63.

16. Paalasmaa, J.; Ranta, M. In Detecting heartbeats in the ballistocardiogram with clustering, Proceedings of the ICML/UAI/COLT 2008 Workshop on Machine Learning for Health-Care Applications, Helsinki, Finland, 2008.

(C) 2014 by the authors; licensee MDPI, Basel, Switzerland. This article is an open access article distributed under the terms and conditions of the Creative Commons Attribution license (http://creativecommons.org/licenses/by/3.0/). 